\title{
DATOS FENOLÓGICOS DE FLORACIÓN DE ALGUNAS ESPECIES LEÑOSAS DE LA DUNA DEL FARO DE TRAFALGAR (BARBATE, CÁDIZ)
}

\author{
María Dolores MORENO DURÁN, José M. ORTEGA \\ y Manuel RODRÍGUEZ DE LOS SANTOS.
}

\begin{abstract}
RESUMEN. Datos fenológicos de floración de algunas especies leñosas de la duna del Faro de Trafalgar (Barbate, Cádiz). En este trabajo se ha estudiado la composición florística y algunos aspectos del comportamiento fenológico de floración de algunas especies del matorral leñoso existente en una duna fija costera del sur de la Península Ibérica. En la comunidad de vegetación leñosa de la duna del Faro de Trafalgar se desarrollan procesos de floración a lo largo de todo el año, observándose un máximo en primavera (Marzo-Abril), que corresponde a la época de mayor disponibilidad de recursos y climatología más favorable. En el verano se produce una regresión en la producción de flores, aunque sin llegar a desaparecer, hasta alcanzarse otro máximo en otoño, aunque de menor magnitud y duración que en primavera. En los meses invernales muchos matorrales presentan floración, hecho por otra parte frecuente en otras comunidades de matorrales mediterráneos.
\end{abstract}

Palabras clave. Floración, plantas leñosas mediterráneas, dunas costeras, España, Andalucía.

SUMMARY. Flowering fenology of some woody species in the Faro de Trafalgar dune (Barbate, Cádiz). We have studied the flower composition and aspects of the fenologic behavior of flowering of some species of the existing woody scrubland in a coastal dune in the south of the Iberian Peninsule. In the woody vegetation community in the Faro de Trafalgar dune flowering processes are developed the whole year, a maximum being observed in spring (March-April) that corresponds to the period of greater availability of resources and favorable climatology. A regression in the production of flowers is produced in the summer, through without getting to disappear, and then another maximum is reached in autumn, although of less magnitude and duration that in spring with time. In the winter months, many scrubs enter flowering, a fact that is frequent in other Mediterranean scrubland communities.

Key words. Flowering, mediterranean woody plants, coastal dunes, Spain, Andalusia.

\section{INTRODUCCIÓN}

Los ritmos cíclicos a que están sometidos todos los seres vivos, y en especial las plantas, constituyen pulsos de actividad cuya intensidad y duración está determinada por factores climáticos, edáficos, geográficos, biológicos o ecológicos propios de cada ecosistema. En las especies vegetales el periodo de floración constituyen una fase de gran valor intrínseco para la continuidad de la propia especie, afectando además al comportamiento 
fenológico del resto de los taxones que componen la comunidad y, por extensión, al conjunto de las relaciones dentro del ecosistema (Mooney et al. 1974, Herrera, 1984, Jordano 1985, Herrera, 1986). Son escasos los trabajos realizados acerca de la floración de los matorrales mediterráneos españoles (Moreno Rodríguez, 1982; Arroyo 1983, 1985 y 1988; Herrera, 1984; Jordano, 1985; Herrera, 1986; Orshan, 1989; Cabezudo et al., 1992 y 1993, entre otros), y especialmente de aquellos que se asientan en cordones de dunas litorales (Laguna et al., 1986). Estudios recientes han puesto de manifiesto que la floración en matorrales mediterráneos presenta máximos primaverales y mínimos estivales, siendo numerosas las especies con largos periodos de floración total, comportándose la comunidad como multiestacional (Cabezudo et al., 1993, Hidalgo y Cabezudo, 1994).

En este trabajo se ha estudiado la composición florística y algunos aspectos del comportamiento fenológico de la floración de algunas especies de plantas leñosas existentes en la duna del Faro de Trafalgar (Barbate, Cádiz).

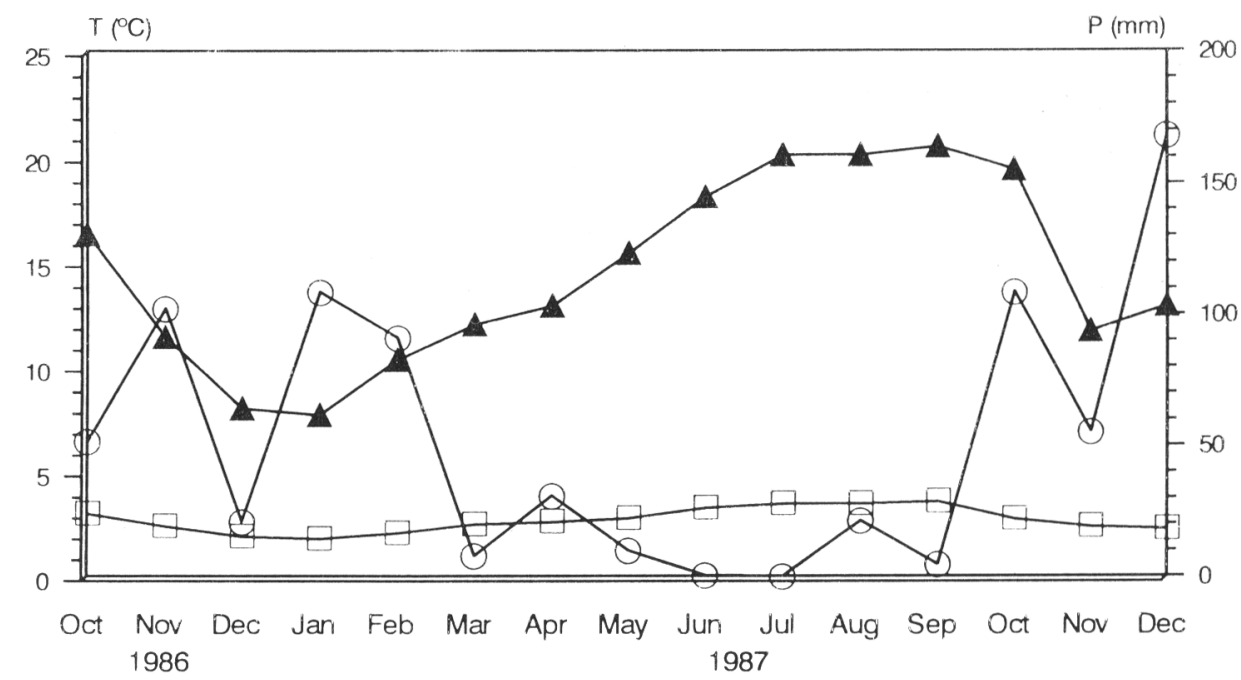

Figura 1. Diagrama ombrotérmico de Barbate (Cádiz), de Octubre de 1986 a Diciembre de 1987. T. mínima: „; T. máxima: $\Delta$; Precipitación: O. Hydrothermic diagram of Barbate (Cádiz), from October 1986 to December 1987.

\section{ÁREA DE ESTUDIO Y MÉTODOS}

El área de estudio se localiza en una antigua duna fija de 1,5 hectáreas de superficie situada en el Faro de Trafalgar (Barbate, Cádiz, $\left.36^{\circ} 16^{\prime} \mathrm{N}, 2^{\circ} 24^{\prime} \mathrm{W}\right)$. El sustrato edáfico original lo constituyen arenas pleistocénicas y calizas con fósiles en las que se asienta una vegetación de características mediterráneas perteneciente a la serie Rhamno-Junipereto macrocarpae del sector corológico Gaditano-OnuboAlgarbiense (Rivas-Martínez, 1982). El clima de la zona es de tipo mediterráneo oceánico (Capel, 1981). Presenta una precipitación media anual de $636 \mathrm{~mm}$ con el 74\% de lluvia caída durante los meses de Septiembre a Febrero y un $26 \%$ en primavera. La temperatura media anual es de $17,5^{\circ} \mathrm{C}$, siendo Enero el mes más frío y Agosto el mes más caluroso, con temperaturas medias de $11,3^{\circ} \mathrm{C}$ y $24,6^{\circ} \mathrm{C}$, respectivamente (fig. 1). La insolación media anual es de alrededor de 3.000 horas de sol.

La vegetación del entorno del Faro de Trafalgar está constituida por taxones pertenecientes a ambientes sabulícolas que contrastan fuertemente con la comunidad de 
matorral que se asienta en la duna, objeto de este estudio (ver Anexo). Este aislamiento respecto a otros matorrales cercanos, de los que dista más de 500 metros, le confiere a sus elementos exigencias ecológicas muy especiales, lo que aún hace más interesante su estudio.

El estudio de la fenología de floración de algunos taxones de plantas leñosas de la zona se llevó a cabo desde Octubre de 1986 a Diciembre de 1987 con una periodicidad mensual, a lo largo de un transecto previamente establecido, que coincidía con un sendero existente, y que recorría la longitud máxima de la duna (125 m., aproximadamente). En cada visita se analizaron todos los individuos existentes en dos metros a un lado y otro del transecto, cuantificándose los individuos de cada especie en floración. Se considera que un individuo esta en floración cuando posee al menos una flor abierta. En este estudio se han seleccionado los taxones mejor representados en el conjunto de la comunidad, descartándose aquellos que presentaban un número de individuos poco significativo en el transecto de muestreo ( $<10$ individuos). El número de individuos muestreados por especie se indica en el Anexo.

\section{RESULTADOS Y DISCUSIÓN}

El número de taxones leñosos presentes en la duna del Faro de Trafalgar es de 20, pertenecientes a 13 Familias de las cuales Cistaceae y Papilionaceae son las mejor representadas (Anexo). De las 20 especies censadas sólo se ha estudiado detalladamente la fenología de floración de 13 de ellas (fig. 2; Anexo). Así, hay especies que florecen en otoño-invierno tales como Juniperus oophora y Juniperus oxycedrus. Otras como Helichrysum picardii presentan floración tan sólo durante el periodo estival. Daphne gnidium, Rosmarinus officinalis y Osyris quadripartita presentan un comportamiento diferente respecto a los otros taxones, al poseer floración durante casi todo el año. Los taxones con una floración fundamentalmente primaveral son Pistacia lentiscus, Rhamnus lycioides, Halimium commutatum, Phillyrea angustifolia, Armeria pungens y Coronilla valentina. Halimium halimifolium presenta floración en primavera y verano. Dado que los patrones fenológicos varían apreciablemente entre años, es necesario resaltar que este estudio se llevó a cabo durante una sola temporada, por lo que algunas de las diferencias encontradas entre nuestros resultados y las de otros autores podrían deberse a la existencia de diferencias climatológicas anuales.

La amplitud del periodo de floración de P. lentiscus es de dos meses (Marzo y Abril), alcanzándose el máximo en el mes de Marzo con algo más del $85 \%$ de los individuos muestreados en flor (fig. 2). Aunque la floración de este taxón es fundamentalmente primaveral, tal como han puesto de manifiesto varios autores (Arroyo, 1983; Herrera, 1984; Herrera, 1986; Hidalgo y Cabezudo, 1994), existen pequeñas variaciones que parecen responder a factores geográficos y/o climáticos. Así, en Israel, $P$. lentiscus posee un segundo periodo floral en otoño que va de Septiembre a Noviembre (Izhaki y Safriel, 1985), mientras que en los jarales de Madrid la floración de esta especie es más temprana, de Enero a Febrero (Moreno Rodríguez, 1982). En Barbate, localidad que dista $3 \mathrm{Km}$ del área de estudio, la floración es más tardía, extendiéndose hasta principios de Mayo (Arroyo, 1983).

Similar patrón de floración presentan $R$. lycioides y $H$. commutatum, con máximos del $93,3 \%$ en Marzo y $76 \%$ en Abril, respectivamente (fig. 2). Algo más corta es la floración de $R$. lycioides en Doñana (Herrera, 1986) con una duración de tan sólo un mes (Abril), mientras que en El Viso (Sevilla) tiene una duración de mes y medio aproximadamente, desde finales de Marzo a principios de Mayo 
Pistacia lentiscus

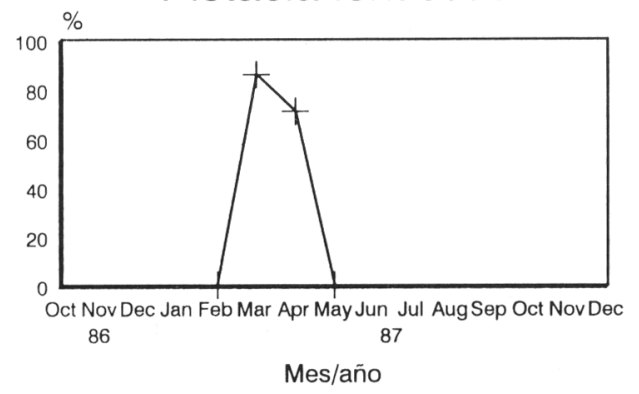

Halimium commutatum

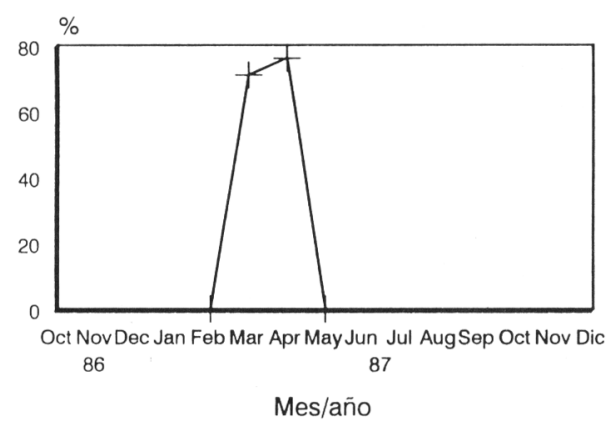

Armeria pungens

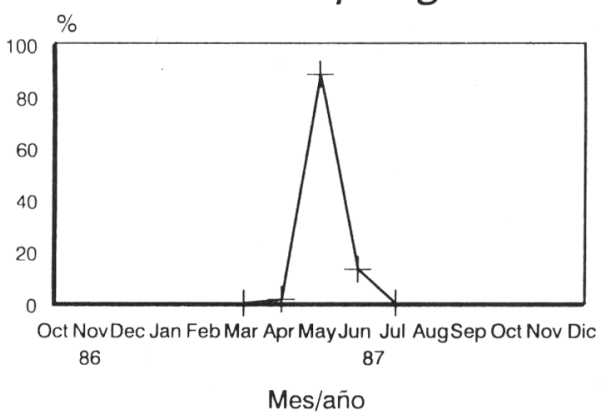

Rhamnus lycioides

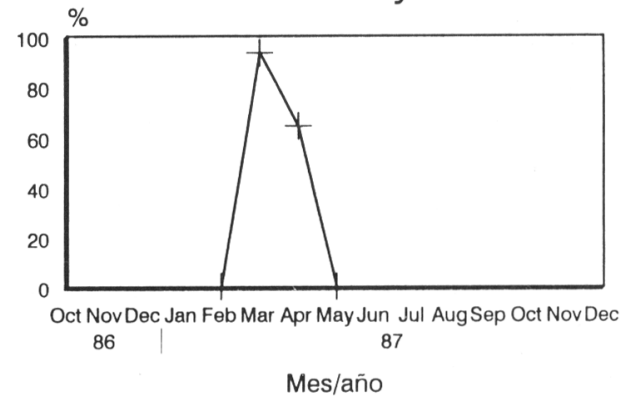

Phillyrea angustifolia

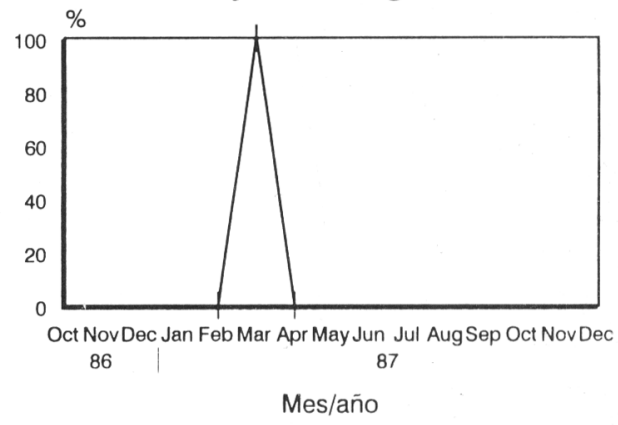

Coronilla valentina

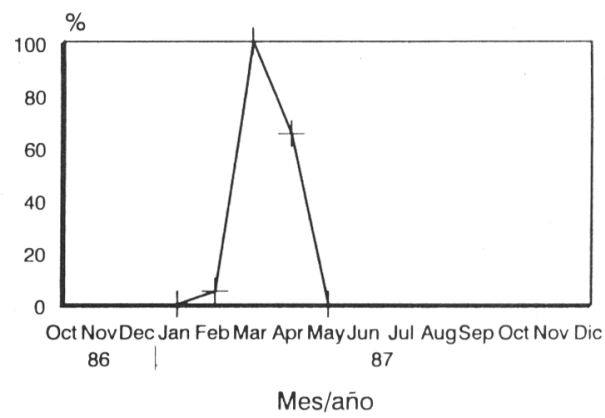

Figura 2. Diagramas fenológicos de floración de las especies leñosas estudiadas en el Faro de Trafalgar (Barbate, Cádiz): + Floración (\%). Phenological diagram of flowering of the schrublans studied in Faro de Trafalgar (Cádiz): + Flowering (\%). 

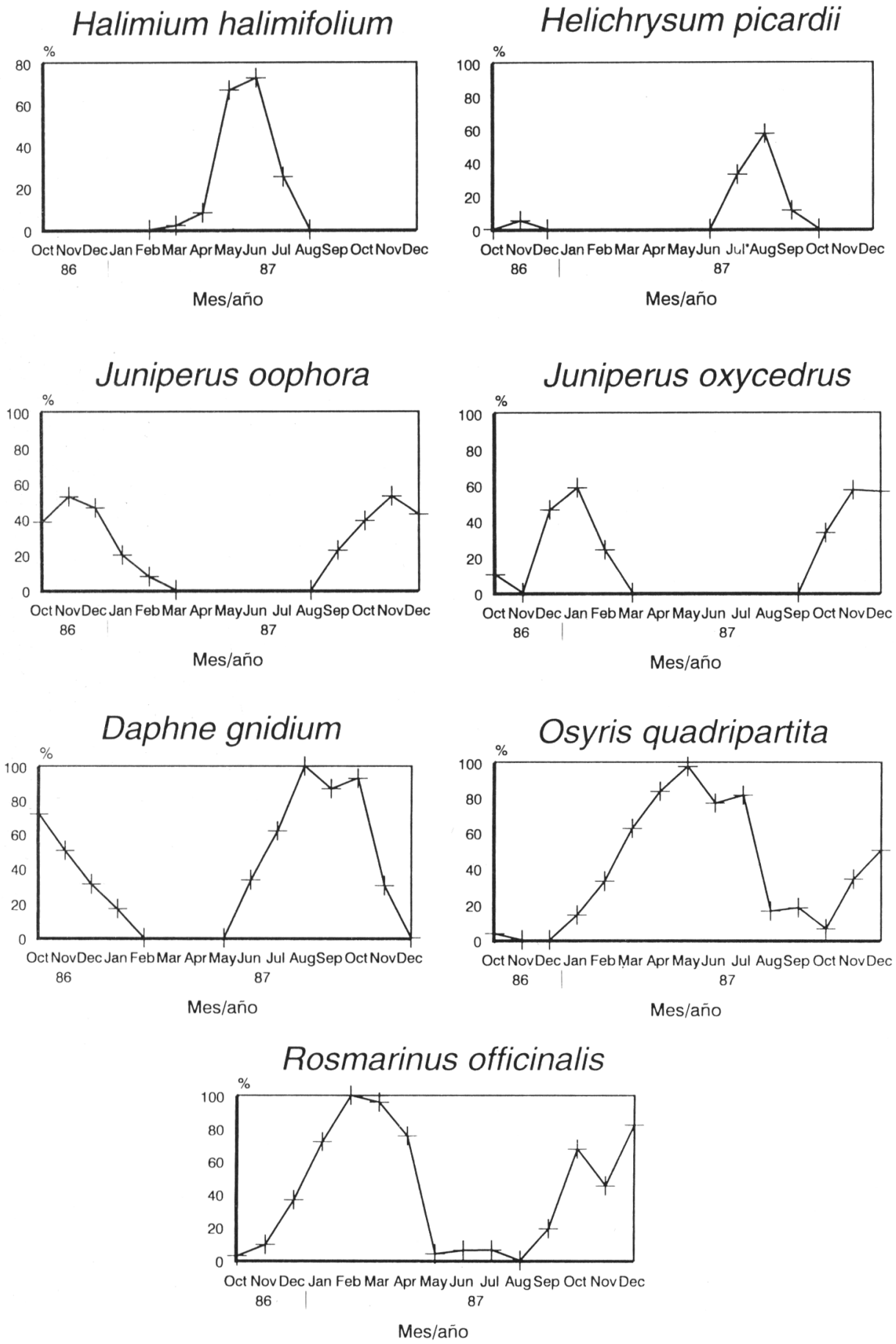

Figura 2. (Continuación) 
(Herrera, 1984). En Barbate la floración de esta especie es más tardía desarrollándose desde mediados de Abril a mediados de Mayo (Arroyo, 1983). Coincidentes resultan los periodos de floración para $H$. commutatum en Barbate (Arroyo, 1983), Doñana (Herrera, 1986) y en el Faro de Trafalgar (este trabajo). En los jarales de Madrid la floración de esta especie es algo más retrasada, y va desde finales del mes de Marzo hasta finales de Mayo (Moreno Rodríguez, 1982).

$P$. angustifolia concentra en el mes de Marzo todo su esfuerzo floral, mes en el que el $100 \%$ de los individuos censados presentan flores (fig. 2). Este tipo de floración es similar a la observada en otras áreas, como Doñana (Herrera, 1986), La Albufera (Laguna et al. 1986), Cazorla y El Viso (Herrera, 1984). En esta última localidad el periodo se extiende desde mediados de Febrero a mediados de Abril; sin embargo, en la Sierra de Mijas (Málaga) el periodo máximo de floración se produce de forma más tardía en el mes de Abril extendiéndose hasta Mayo (Hidalgo y Cabezudo, 1994). En los Montes de Málaga esta especie tiene la floración desde Marzo a Abril (Cabezudo et al., 1993). A. plingens posee también un periodo de floración relativamente corto, de sólo 2 meses, observándose el máximo $(88,2 \%)$ en Mayo (fig. 2). El periodo de floración más largo de las especies primaverales corresponde a $C$. valentina, que posee flores durante 3 meses, observándose el máximo (100\%) en el mes de Marzo (fig. 2).

Aunque su máximo se sitúa al final de primavera y en verano $(72,5 \%$ en Junio), $H$. halimifolium posee un periodo de floración relativamente largo (5 meses), que va desde principios de primavera hasta mediados de verano (fig. 2). Este periodo es muy similar al observado en otras áreas de la península (Arroyo 1983, Laguna et al. 1986, Herrera, 1986, Hidalgo y Cabezudo, 1994). H. picardii presenta los valores más altos de floración durante el estío, con un $57 \%$ de individuos en flor en Agosto, y un periodo total de duración de 3 meses (fig. 2). Esta floración resulta algo más tardía respecto a la observada para esta especie en Barbate (Arroyo, 1983) y Doñana (Herrera, 1986), donde los máximos se observan en Julio.

Los taxones $J$. oophora y $J$. oxycedrus presentan una floración exclusivamente otoñoinvernal, con una duración de 6 meses para el primero y de 5 para el segundo (fig. 2). En esta época la climatología no favorece la presencia de elementos florales, lo que podría explicar que el máximo de individuos con flores no haya superado nunca el $60 \%$ de los muestreados. Otra posible interpretación es que parte de los individuos florecieron durante el año de estudio y otros no, sistema de floración por otra parte característico del género Juniperus que tiende a alternar las floraciones en periodos bianuales. En la Sierra de Mijas el periodo de floración de $J$. oxycedrus tiene una duración de tan sólo 3 meses, desde Octubre a Diciembre (Hidalgo y Cabezudo, 1994). En los Montes de Málaga la floración de esta especie contrasta con las demás localidades comparadas en este trabajo, pues se produce entre los meses de Marzo y Abril (Cabezudo et al., 1993).

El grupo de especies constituido por $D$. gnidium, $O$. quadripartita y $R$. officinalis presentan un comportamiento diferente respecto al resto de los taxones. Poseen un periodo de floración que se extiende a lo largo de la mayor parte del año, aunque con máximos claramente estacionales. Así, D. gnidium parece presentar un periodo floral estival y otoñoinvernal, con una interrupción del proceso desde Febrero hasta Mayo (fig. 2), comportamiento similar al observado en las Sierras de Mijas y en los Montes de Málaga (Hidalgo y Cabezudo, 1994, Cabezudo et al., 1993, respectivamente). El máximo de floración se observa en Agosto. Esta especie posee un periodo de floración más corto en otras áreas como Doñana (Herrera, 1986) o El Viso (Herrera, 1984). 
O. quadripartita muestra el máximo de floración en el mes de Mayo (97,5\%) y presenta una floración circum-anual, con una disminución del proceso, aunque sin llegar a interrumpirse, a finales de verano y mediados de otoño (fig. 2). Extremadamente corto (un mes y medio) resulta su periodo de floración en La Albufera y Barbate (Laguna et al., 1986, Arroyo, 1983). Sin embargo, es más extenso en Doñana (Herrera, 1986) y El Viso (Herrera, 1984) con 6 y 9 meses respectivamente, aunque siempre de menor duración a la observada en nuestra zona. Al igual que el taxón anterior, $R$. officinalis posee un periodo de floración que se extiende durante casi todo el año, situándose los máximos desde mediados de invierno a mediados de primavera (fig. 2). Desde esa época hasta Septiembre, el proceso disminuye drásticamente para alcanzar nuevamente valores altos en otoño e invierno. Es en este taxón donde existen mayores diferencias en los periodos florales entre diferentes áreas mediterráneas, fluctuando entre tres meses, de Marzo a Junio en La Albufera (Laguna et al., 1986), de Febrero a Mayo en Madrid (Moreno Rodríguez, 1982) y de Marzo a Mayo en Barbate (Arroyo, 1983), los casi 5 meses en Doñana, desde Diciembre a mediados de Abril (Herrera, 1986), los 6 meses en la Sierra de Mijas de Octubre a Marzo (Hidalgo y Cabezudo, 1993), hasta los 10 meses en Tabernas (Almería), de Octubre a Julio (Navarro et al., 1993). En ningún caso se supera el periodo de floración observado en el Faro de Trafalgar.

En la comunidad de especies de plantas de matorral estudiada en la duna del Faro de Trafalgar se desarrollan procesos de floración a lo largo de todo el año, observándose un máximo en primavera (Marzo-Abril, fig. 3), que corresponde a la época de mayor disponibilidad de recursos y climatología más favorable. Este sistema de floración es similar al observado para el matorral en otros ecosistemas mediterráneos (Mooney et al., 1974; Kummerov, 1983; Herrera, 1984; Reader, 1984; Herrera, 1986; Cabezudo et al., 1993; Navarro et al., 1993; Hidalgo y Cabezudo, 1994). En el verano se produce una regresión en la producción de flores, aunque sin llegar a desaparecer, desarrollándose otra floración en otoño, tras la caída de las primeras lluvias (Septiembre a Noviembre), aunque de menor magnitud y duración que en primavera. En los meses invernales muchos matorrales presentan

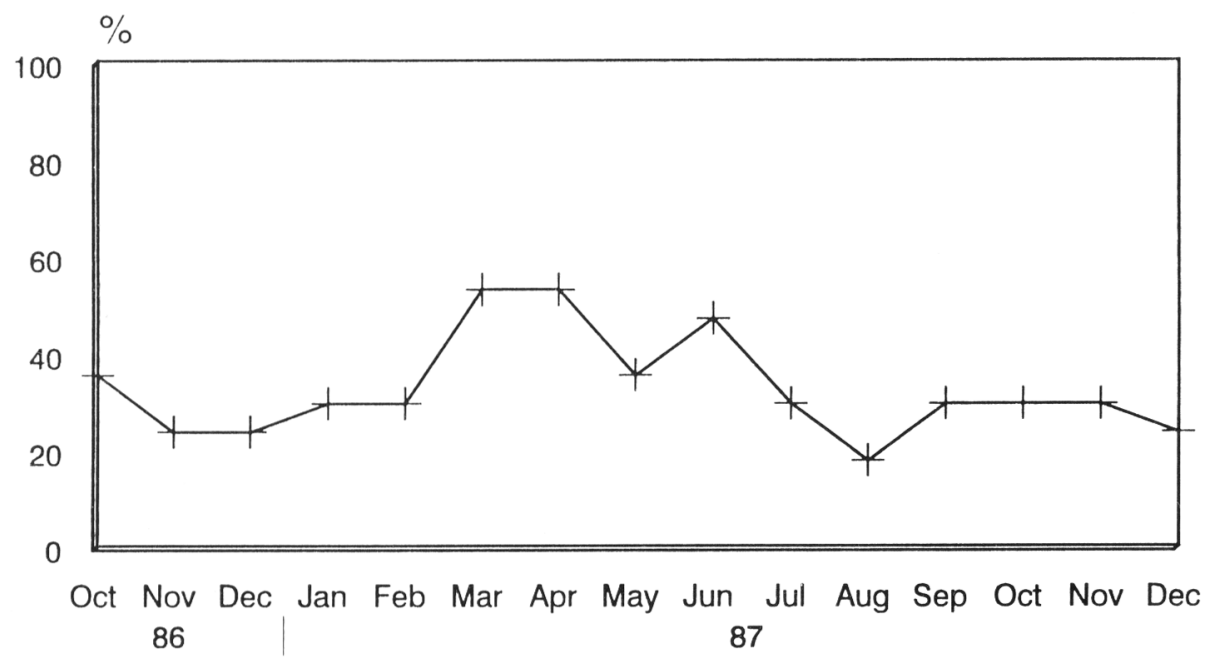

Figura 3. Porcentaje mensual de especies en la fenofase de floración. Monthly percentage of species showing flowering phenophase. 
floración, hecho por otra parte frecuente en otras comunidades de matorrales mediterráneos (Reader; 1984; Herrera, 1986). El máximo de floración se alcanza en los meses de Marzo y Abril, cuando más del $52 \%$ de las especies de matorral presentan floración. Aunque la floración aparece durante todo el año, el pico principal, de Marzo a Junio, ocupa aproximadamente unos 4 meses, mientras que el segundo pico (Septiembre-Noviembre) ocupa 3 meses con casi un $30 \%$ de las especies consideradas. Los meses con mayor número de especies en floración (9) fueron Marzo y Abril, meses donde florece en ambos casos el $53 \%$ de las especies estudiadas. Agosto es el mes con menor número de especies en floración con tan solo 3 (17\%); en el resto de los meses no bajaron del $23 \%$ de las especies consideradas. El óptimo de floración en primavera está favorecido por la buena disponibilidad de agua en el suelo debido a las precipitaciones caídas durante los meses anteriores, la suavidad de las temperaturas y la alta actividad de los insectos, entre otros factores (Mooney et al., 1974; Baker et al,. 1982; Kummerov, 1983). Después de la pausa veraniega, en la que la floración disminuye en más de un $35 \%$, se observa un aumento paulatino del porcentaje de flores en otoño llegando hasta casi un $30 \%$. Esto parece concordar con una distribución bimodal de floración, comportamiento similar al observado en parcelas próximas a la estudiada (Arroyo, 1983), y en otras áreas del sur peninsular de parecidas características ecológicas (Herrera, 1986, Hidalgo y Cabezudo, 1994).

El 76,5\% de las especies estudiadas en el Faro de Trafalgar tienen polinización entomófila. La actividad de los insectos durante el invierno en ecosistemas mediterráneos es un hecho descrito por varios autores (Herrera, 1978, 1980), especialmente en áreas cercanas al mar, donde la benignidad y suavidad de las temperaturas junto con la gran insolación favorece la presencia de polinizadores (Herrera,
1988), permitiendo así la existencia de una floración invernal relativamente importante.

\section{ANEXO}

Taxones leñosos presentes en la duna del Faro de Trafalgar (las especies estudiadas en este trabajo vienen marcadas con una cruz; entre paréntesis se indica el número de individuos muestreados para cada especie).

\section{Anarcadiaceae \\ + Pistacia lentiscus L. (40).}

\section{Cistaceae}

Cistus salvifolius L.

+ Halimium commutatum Pau (45).

+ Halimium halimifolium (L.) Willk. (50).

\section{Compositae}

Anthemis maritima L.

+ Helichrysum picardii Boiss. \& Reuter (150).

\section{Cupressaceae}

+ Juniperus oophora G. Kunze (73).

+ Juniperus oxycedrus subsp. macrocarpa (Sibth. \& Sm.) Ball (22).

\section{Labiatae}

+ Rosmarinus officinalis L. (80).

Teucrium lusitanicum Schreber

\section{Oleaceae}

+ Phillyrea angustifolia L. (25).

\section{Palmae}

Chamaerops humilis L.

\section{Papilionaceae}

+ Coronilla valentina subsp. glauca (L.) Batt. (35). Lotus creticus L.

Ononis cossoniana Boiss. \& Reuter

\section{Plumbaginaceae}

+ Armeria pungens (Link) Hoffmanns. \& Link (80).

\section{Rhamnaceae}

+ Rhamnus lycioides L. subsp. lycioides (25). 


\section{Rutaceae}

Ruta angustifolia Peers.

\section{Santalaceae}

+ Osyris quadripartita Salzm. ex Decne (40).

\section{Thymeleaceae}

+ Daphne gnidium L. (25).

AGRADECIMIENTOS. Agradecemos a Isabel Durán su apoyo y compañía durante los días de muestreo. Así mismo, a $\mathrm{M}^{\mathrm{a}}$ Teresa Díez por la traducción al inglés del resumen.

\section{BIBLIOGRAFÍA}

ARROYO, J. -1983- Algunos aspectos fenológicos de la floración del matorral en Andalucía Occidental. En: Avances sobre la Investigación en Bioclimatología. CSIC-Univ. Salamanca, pp. 263-272.

ARROYO, J. -1985-Fenología de la floración en matorrales de Andalucía Occidental. Tesis Doctoral. Universidad de Sevilla.

ARROYO, J. -1988- Atributos florales y fenología de la floración en matorrales del sur de España. Lagascalia 1(1): 43-78.

BAKER, G.A., P.W. RUNDEL \& D.J. PARSONS 1982- Comparative phenology and growth in three chaparral shrubs. Bot. Gaz. 143: 94-100.

CABEZUdo, B., T. NAVARRO, A.V. PÉREZ LATORRE, J.M. NIETO CALDERA y G. ORSHAN -1992-Estudios fenomorfológicos en la vegetación del sur de España. I. Cistus. Acta Bot. Malacitana 17: 229-237.

CABEZUdo, B., A.V. PÉrez latorre, T. NAVARRO y J.M. NIETO CALDERA -1993Estudios fenomorfológicos en la vegetación del sur de España. II. Alcornocales Mesomediterráneos. (Montes de Málaga, Málaga). Acta Bot. Malacitana 18: 179-188.

CAPEL, J.J. -1981- Los climas de España. Ed. Oikos-Tau. Barcelona.
HERRERA, C.M. -1978- Ecological correlates of residence and no residence in a mediterranean passerine bird community. J. Anim. Ecol. 47: 871-890.

HERRERA, C.M. -1980- Composición y estructura de dos comunidades mediterráneas de passeriformes. Doñana, Acta Vert. 7: 1-340.

HERRERA, C.M. -1984- A study of avian frugivores, bird- dispersed plants and their interactions in Mediterranean scrublands. Ecol. Monogr. 54: 1-23.

HERRERA, J. -1986- Flowering and fruiting phenology in the coastal shrublands of Doñana, south Spain. Vegetatio 68: 91- 98.

HERRERA, J. -1988- Pollination relationships in southern spanish mediterranean shrublands. $J$. Ecol. 76: 274-287.

HIDALGO, M.I. y B. CABEZUDO - 1994- Fenología y volumen de floración del matorral de la Sierra de Mijas (Málaga, S. España). Acta Bot. Malacitana 19: 123-136.

IZHAKI, I \& U.N. SAFRIEL - 1985- Why do fleshyfruit plants of the mediterranean scrub intercept fall- but not spring- passage of seed-dispersing migratory birds?. Oecologia (Berlin) 67: 40-43. JORDANO, P. -1985- El ciclo anual de los paseriformes frugívoros en el matorral mediterráneo del sur de España: importancia de su invernada y variaciones interanuales. Ardeola 32(1): 69-94.

KUMMEROV, J. -1983-Comparative phenology of Mediterranean- type plant communities. In: F.J. Kruger, D.T. Mitchell y J.U.M. Jarvis (ed.), Ecological Studies 43: Mediterranean- type ecosystems, pp. 300-317. Springer, New York.

LAGUNA, E., M. GUARA y E. SANCHÍZ -1986Estudios ecológicos de un transecto dunal. II. Fenología. Fol. Bot. Misc. 5: 105-116.

MOONEY, H.A., D.J. PARSONS \& J. KUMMEROV -1974- Plant development in Mediterranean climates. In: H. Lieth (ed.), Phenology and seasonality modeling, pp. 255-267. Springer, New York. 
MORENO RODRÍGUEZ, J.M. -1982- Estudios ecológicos en jarales (Cistion laurifolii). Variación anual del entorno y algunas manifestaciones fenológicas. Fundación Juan March. Monog. Serie Universitaria $\mathrm{n}^{\circ} 180$.

NAVARRO, T., J.M. NIETO CALDERA, A.V. PÉREZ LATORRE y B. CABEZUDO -1993Estudios fenomorfológicos en la vegetación del sur de España. III. Comportamiento estacional de una comunidad de badlands (Tabernas, Almería. España). Acta Bot. Malacitana 18: 189-198.

ORSHAN, G. -1989- Plant phenomorphological studies in Mediterranean type ecosystems. Kluwer Academic Publishers. Dordrecht.

READER, R.J. -1984- Comparison of the annual flowering schedules for Scottish heathland and mediterranean-type shrublands. Oikos 43: 1-8.
RIVAS-MARTÍNEZ, S. -1982- Etages bioclimatiques, secteurs corologiques et séries de végétation de l'Espagne méditerranéenne. Ecologia Mediterranea 8: 275-288.

Aceptado para su publicación en Mayo de 1997

Dirección de los autores. M.D. MORENODURÁN: I.E.S. Santa Olalla del Cala, Av. de Extremadura s/n, Santa Olalla del Cala (Huelva). J.M. ORTEGA: Instituto de Bioquímica Vegetal y Fotosíntesis, Universidad de Sevilla y CSIC, Centro de Investigaciones Científicas «Isla de la Cartuja», Av. Americo Vespucio s/n, 41092-Sevilla. M. RODRÍGUEZ DE LOS SANTOS: Dpto. de Protección de Fauna y Flora. Consejería de Medio Ambiente. Pabellón de Nueva Zelanda. Isla de la Cartuja. 41071-Sevilla. 